\title{
18F-FDG PET/CT and cardiac CTA in transcatheter aortic valve implanted endocarditis: Still at the beginning of a long road
}

\author{
Albert Roque, $M D,^{\mathrm{a}, \mathrm{c}, \mathrm{d}, \mathrm{e}}$ and María Nazarena Pizzi, $M D, \mathrm{PhD}^{\mathrm{b}, \mathrm{d}, \mathrm{e}}$ \\ a Department of Radiology, Hospital Universitari Vall d'Hebron, Barcelona, Spain \\ b Department of Cardiology, Hospital Universitari Vall d'Hebron, Barcelona, Spain \\ c IDI (Institut de Diagnòstic per la Imatge), Barcelona, Spain \\ d Universitat Autònoma de Barcelona, Barcelona, Spain \\ e Vall d'Hebron Institut de Recerca (VHIR), Barcelona, Spain
}

Received Dec 20, 2019; accepted Dec 22, 2019

doi: $10.1007 / \mathrm{s} 12350-020-02035-1$

\section{See related article, pp. 2072-2082}

Despite constant advances in the diagnosis and treatment of infective endocarditis (IE), it remains a serious disease, often associated with a poor prognosis. The diagnosis of IE is challenging, especially in patients with prosthetic valves, cardiac implantable electronic devices, and other intracardiac prosthetic material. Difficulties in the interpretation of echocardiographic studies in patients with prosthetic valves limit the diagnostic performance of the modified Duke criteria (DC) in prosthetic valve endocarditis (PVE). 18F-Fluorodeoxyglucose (18F-FDG) positron emission tomography/computed tomography (PET/CT) has proven useful in patients with suspected PVE, improving the diagnostic accuracy when added to DC. Hence, PET/ CT has become a major criterion in the European Society of Cardiology (ESC) 2015 diagnostic criteria for PVE. ${ }^{1-3}$ In addition, cardiac computed tomography angiography (CTA) which may be superior to echocardiography for depicting perivalvular complications in $\mathrm{IE},{ }^{4,5}$ is also considered a major imaging criterion and is recommended in the diagnostic algorithm of both native and prosthetic valve IE.

Transcatheter aortic valve implantation (TAVI) is becoming an increasingly more common procedure

Reprint requests: María Nazarena Pizzi, MD, PhD, Department of Cardiology, Hospital Universitari Vall d'Hebron, Barcelona, Spain; nachi_pizzi@yahoo.com.ar

J Nucl Cardiol 2021;28:2083-5.

1071-3581/\$34.00

Copyright (C) 2020 American Society of Nuclear Cardiology. worldwide. ${ }^{6,7}$ The endocarditis rates after TAVI (TAVIIE) do not differ significantly from those reported for surgical aortic valve replacement. ${ }^{8,9}$ Thus, the population of patients undergoing TAVI is increasing, and among suspected PVE, the number of patients with TAVI-IE is rising, with their additional diagnostic difficulties, as echocardiographic images may be even more difficult to interpret in these cases. Based on these considerations, it seems reasonable that a multimodality and multidisciplinary approach will offer the greatest diagnostic benefits in this growing clinical setting. ${ }^{10}$ Within this strategy, 18F-FDG PET/CT and cardiac CTA may play a key role in the diagnostic algorithm for TAVI-IE. However, although the diagnostic yield of these techniques has been demonstrated in PVE related to surgically implanted prosthetic valves, there is little data about the diagnostic performance in TAVI-IE beyond a few case reports and a brief series of 16 patients. $^{10,11}$

In this issue of Journal of Nuclear Cardiology, Wahadat et al $^{14}$ aimed to assess the additional diagnostic value of 18F-FDG PET/CT and cardiac CTA in suspected TAVI-IE when added to the modified DC. In their multicenter retrospective analysis, the authors evaluated 30 patients with suspected TAVI-IE who underwent 18F-FDG PET/CT and/or cardiac CTA. Patients were initially classified as having a definite, possible, or rejected TAVI-IE diagnosis based on the modified DC and then were reclassified after adding the 18F-FDG PET/CT and/or cardiac CTA information using the ESC criteria. The authors found that the imaging data led to a change in the final diagnosis (established by consensus of the Endocarditis Team) in $33 \%$ of patients. Nonetheless, certain aspects related to 
the methods and the authors' affirmations merit discussion.

First, although the encouraging results seem to show that the diagnostic yield of 18F-FDG PET/CT and cardiac CTA observed in "conventional" PVE can also apply to TAVI-IE, the relatively small number of patients in the study, its retrospective nature, and the heterogeneity of the group (including different types of TAVI), limits generalization of the results. In addition, there was a considerable imbalance between the number of patients evaluated by $18 \mathrm{~F}-\mathrm{FDG}$ PET/CT and those assessed by CTA. The latter technique was used almost exclusively in the definite group, which hardly allows conclusions about cardiac CTA to be derived from the study. (Perhaps it should have focused only on the role of PET/CT). A prospective study with a larger number of patients will be needed to verify the reproducibility of the findings and determine the usefulness of these techniques in the TAVI-IE population, especially cardiac CTA.

The combined ability of 18F-FDG PET/CT to detect inflammation/infection and CTA to define structural damage has proven to provide the highest diagnostic yield in PVE. ${ }^{3}$ In the study by Wahadat et al, ${ }^{12}$ 18F-FDG PET/CT and CTA were performed separately, with no clear description of the equipment, protocols, or time gap between scans. This approach might miss the synergistic advantage provided by combining advanced cardiac images acquired simultaneously in a hybrid examination: (1) As IE is a rapidly progressive condition, the related lesions can change in a very short time. In this sense, acquisition of metabolic and the anatomic images at the same time point of the disease is important for a concordant diagnosis and (2) Hypermetabolism is consistent with infection in most cases, but sometimes 18F-FDG uptake may be difficult to interpret and increased uptake, in itself, does not always imply infection. Therefore, a direct correlation of the metabolic information with the anatomic findings can be the key to achieving a definite diagnosis when reading 18F-FDG PET/CT images. ${ }^{13,14}$

Proper patient preparation is essential for cardiac 18F-FDG PET/CT because significant physiological myocardial 18F-FDG uptake can lead to false-positive or false-negative diagnoses. For this reason, the recommendations are very clear about achieving myocardial suppression, basically by following a highfat and low-carbohydrate diet combined with a long fasting period. ${ }^{15}$ The rate of myocardial suppression reported in the series under discussion is unclear; hence, the results obtained may also have been affected due to this reason.

Another issue yet to be resolved, invariably linked to nuclear medicine techniques, is the constant aspiration to have cut-off values to rule in or rule out a diagnosis. However, standardized acquisition and quantification, essential for generalizing threshold values, remains to be resolved. Up to now, there is no agreement among centers and vendors, and although reconstruction algorithms have been proposed by European Association of Nuclear Medicine (EANM) Research Ltd. (EARL), they have not proven superior to modern iterative reconstruction methods incorporating time-of-flight and other parameters. ${ }^{16}$ Hence, EARL reconstructions can be used, but they are not formally recommended or validated for cardiac purposes, and they lead to relatively high smoothing of the images, resulting in lower SUVmax values. Furthermore, many centers do not have EANM accreditation and this represents another impediment for quantitative results. As would be expected, Wahadat et al $^{12}$ did not find significant differences between values obtained with EARL and non-EARL reconstructions. Furthermore, as $18 \mathrm{~F}$ FDG PET/CT studies were classified as positive or negative according to visual analysis, it seems that quantitative information was not considered a part of the diagnostic criteria.

In conclusion, as has been demonstrated for PVE related to surgically implanted prostheses, a multimodality imaging approach including 18F-FDG PET/CT and CTA seems to be the best strategy to progressively improve the diagnosis and, hopefully, the prognosis of TAVI-IE patients. Suspected TAVI-IE is becoming increasingly more common, and it involves complex, virtually inoperable patients with multiple comorbidities. This relatively new clinical scenario is also a challenge for clinical management. The exact type and duration of conservative treatment, mainly prolonged antibiotic therapy, has yet to be determined. As surgery is only a remote option, it would be desirable to reproduce the good diagnostic yield obtained by $18 \mathrm{~F}-\mathrm{FDG}$ PET/CT and CTA in PVE, as these techniques will surely play a fundamental role in decision-making for the diagnosis and management of TAVI-IE.

\section{Disclosure}

Albert Roque and María Nazarena Pizzi declare that they have no conflict of interest.

\section{References}

1. Habib G, Lancellotti P, Antunes MJ, Bongiorni MG, Casalta JP, Del Zotti F, et al. ESC Guidelines for the management of infective endocarditis. The Task Force for the Management of Infective Endocarditis of the European Society of Cardiology (ESC). Endorsed by: European Association for Cardio-Thoracic Surgery 
(EACTS) and the European Association of Nuclear Medicine (EANM). Eur Heart J 2015;36:3075-4023.

2. Saby L, Laas O, Habib G, Cammilleri S, Mancini J, Tessonnier L, et al. Positron emission tomography/computed tomography for diagnosis of prosthetic valve endocarditis increased valvular $18 \mathrm{~F}$ fluorodeoxyglucose uptake as a novel major criterion. J Am Coll Cardiol 2013;61:2374-82.

3. Pizzi MN, Roque A, Fernández-Hidalgo N, Cuéllar-Calabria $H$, Ferreira-González I, Gonzàlez-Alujas MT, et al. Improving the diagnosis of infective endocarditis in prosthetic valves and intracardiac devices with 18F-FDG-PET/CT-angiography: Initial results at an infective endocarditis referral center. Circulation 2015;132:1113-26.

4. Entrikin DW, Gupta P, Kon ND, Carr JJ. Imaging of infective endocarditis with cardiac CT angiography. J Cardiovasc Comput Tomogr 2012;6:399-405.

5. Feuchtner GM, Stolzmann P, Dichtl W, Schertler T, Bonatti J, Scheffel H, et al. Multislice computed tomography in infective endocarditis: Comparison with transesophageal echocardiography and intraoperative findings. J Am Coll Cardiol 2009;53:436-44.

6. Smith CR, Leon MB, Mack MJ, Miller DC, Moses JW, Svensson LG, PARTNER Trial Investigators, et al. Transcatheter versus surgical aortic-valve replacement in high-risk patients. N Engl J Med 2011;364:2187-98.

7. Makkar RR, Fontana GP, Kapadia S, Pichard AD, Douglas PS, Thourani VH, et al. Transcatheter aortic-valve replacement for inoperable severe aortic stenosis. N Engl J Med 2012;366:1696704.

8. Thomas M, Schymik G, Walther T, Himbert D, Lefèvre T, Treede $\mathrm{H}$, et al. One-year outcomes of cohort 1 in the Edwards SAPIEN Aortic Bioprosthesis European Outcome (SOURCE) registry: The European registry of transcatheter aortic valve implantation using the Edwards SAPIEN valve. Circulation 2011;124:425-33.

9. Butt JH, Ihlemann N, De Backer O, Søndergaard L, HaversBorgersen E, Gislason GH, et al. Long-term risk of infective endocarditis after transcatheter aortic valve replacement. J Am Coll Cardiol 2019;73:1646-55.

10. Salaun E, Sportouch L, Barral PA, Hubert S, Lavoute C, Casalta AC, et al. Diagnosis of infective endocarditis after TAVR: Value of a multimodality imaging approach. JACC Cardiovasc Imaging 2018;11:143-6.

11. Swart LE, Scholtens AM, Liesting C, van Mieghem NM, Krestin GP, Roos-Hesselink JW, et al. Serial 18F-fluorodeoxyglucose positron emission tomography/CT angiography in transcatheterimplanted aortic valve endocarditis. Eur Heart J 2016;37:3059.

12. Wahadat Ali R, Tanis W, Swart Laurens E, Scholtens A, Krestin GP, van Mieghem NMDA, et al. Added value of 18F-FDG-PET/ $\mathrm{CT}$ and cardiac CTA in suspected transcatheter aortic valve endocaritis. J Nucl Cardiol 2019. https://doi.org/10.1007/s12350019-01963-х.

13. Pizzi MN, Roque A, Cuéllar-Calabria $\mathrm{H}$, Fernández-Hidalgo $\mathrm{N}$, Ferreira-González I, González-Alujas MT, et al. Infective versus inflammatory patterns in 18F-FDG-PET/CTA of prosthetic cardiac valves and valve-tube grafts. J Am Coll Cardiol Imaging 2016;9:1224-7.

14. Roque A, Pizzi MN, Fernández-Hidalgo N, Permanyer E, CuellarCalabria H, Romero-Farina G, et al. Morpho-metabolic post-surgical patterns of non-infected prosthetic heart valves by [18F]FDG PET/CTA: "Normality" is a possible diagnosis. Eur Heart J Cardiovasc Imaging 2020;21:24-33.

15. Erba PA, Lancellotti P, Vilacosta I, Gaemperli O, Rouzet F, Hacker M, et al. Recommendations on nuclear and multimodality imaging in IE and CIED infections. Eur J Nucl Med Mol Imaging 2018;45:1795-815.

16. Kaalep A, Sera T, Rijnsdorp S, Yaqub M, Talsma A, Lodge MA, et al. Feasibility of state of the art PET/CT systems performance harmonisation. Eur J Nucl Med Mol Imaging 2018;45:1344-61.

Publisher's Note Springer Nature remains neutral with regard to jurisdictional claims in published maps and institutional affiliations. 